\title{
EFECTO DE LA EXTRACCIÓN DEL ALGA Lessonia trabeculata, VILLOUTA \& SANTELICES (1986), SOBRE EL MACROBENTOS EN MARCONA, PERÚ.
}

\author{
Douglas Vera-Izurieta ${ }^{1,2}$, Diana Quinteros $\operatorname{Carlos}^{1}$, Ma. Herminia Cornejo-Rodríguez ${ }^{2}$, Susan Donayre Salazar \\ ${ }^{1}$ Universidad Nacional Agraria La Molina (UNALM). \\ ${ }^{2}$ Universidad Estatal Península de Santa Elena (UPSE). \\ ${ }^{3}$ Instituto del Mar del Perú (IMARPE). \\ E-mail: $\underline{\text { douglas } 23 \text { blgo@yahoo.es }}$
}

\begin{abstract}
RESUMEN
Las macroalgas son organismos dominantes en cobertura y biomasa en mares fríos y templados de las costas rocosas de todo el mundo. La sobreexplotación del recurso pone en riesgo dos aspectos muy importantes: 1) se está permitiendo que no haya una renovación apropiada de las praderas naturales y 2) se estaría afectando a las comunidades del macrobentos. En este sentido el presente trabajo determinó el efecto de la extracción de Lessonia trabeculata sobre la riqueza y abundancia del macrobentos asociado a esta alga en Marcona. Se realizó un muestreo en 02 sectores (Lobo Fino y Basural), en cada uno de los cuales se ubicaron 03 transectos con 03 estaciones (replicas), distribuidos en intervalos de profundidad de: 5-10 m, $10-15 \mathrm{~m}$ y $15-20 \mathrm{~m}$, obteniendo en total 18 discos de adhesión y 9 interdisco. Se registraron un total de 5399 organismos agrupados en 71 especies distintas, identificándose 5369, correspondiendo 31 especies al Phylum Anélida, 17 a Mollusca, 15 a Crustácea, 4 a Equinodermata, 1 a Tunicata, 1 a Nemertea, 1 a Picnogónida y 1 a la Ictofauna del sector. La Riqueza de especies (S) en las muestras intradisco varió entre 13 y 37 siendo las subestaciones P.3.2 y P.3.3 en Lobo Fino con valores más altos, mientras en las muestras interdisco la $S$ evidenciada varía de 8 a 24, con el mayor registro en la estación I.4 del Basural. El análisis de clúster realizado con el PRIMER 6.0 nos da como resultado que entre las subestaciones de El Basural existe una similaridad del $61 \%$ siendo la subestación B.4.3 la que se diferencia en un solo grupo por la mayor $S$ de poliquetos y entre las subestaciones de Lobo Fino a un $62 \%$ las subestaciones L.1.1 y L.1.2 forman grupos separados por las diferencias existentes en la diversidad de moluscos y crustáceos.
\end{abstract}

Palabras claves: Macroalgas, macrobentos, discos de adhesión.

\begin{abstract}
Macroalgae are dominant organisms in cover and biomass in cold and temperate seas of the rocky shores worldwide. The overexploitation of the resource endangers two important aspects: 1) it is allowing no proper renewal of natural pastures and 2) be affecting macrobenthic communities. The aim of this work was determined the effect of the removal Lessonia trabeculata on the richness and abundance of macrobenthos associated to algae in Marcona. Sampling in 02 sectors (Lobo Fino and Basural), in each 03 transects with 03 stations distributed in depth intervals were located was performed: 5-10 m, 10-15 m and 15-20 m, obtaining a total of 18 holdfasts as samples and 9 between disk. A total of 5399 organisms grouped in 71 different species were recorded, identifying 5369, corresponding to 31 species of Phylum Annelida, 17 to Mollusca, 15 to Crustacea, 4 to Equinodermata, 1 to Tunicata, 1 to Nemertea, 1 to the Pycnogonida and 1 to the ichthyofauna of the sector. The species richness $(S)$ on the discs varied between 13 and 37 being the P.3.2 and P.3.3 substations of Lobo Fino with higher values, while among the $S$ evidenced between disks varies from 8 to 24 , with higher registration of I.4 station of Basural. The cluster analysis performed with PRIMER 6.0 gives us the result that between the substations of Basural exists a similarity of $61 \%$ being B.4.3 substation which differs in one group the highest species richness of polychaetes and between substations of Lobo Fino to 62\% L.1.1 and L.1.2 substations are separated by differences in diversity of molluscs and crustaceans.
\end{abstract}

Keywords: Macroalgae, macrobenthos, holdfasts. 


\section{Introducción}

En el litoral centro-sur peruano, los bancos naturales de recursos bentónicos de fondos duros presentan una amplia biodiversidad, que incluye un importante número de especies de invertebrados y macroalgas de interés comercial. Estos son extraídos y recolectados por las comunidades de pescadores artesanales localizados en toda la costa de las regiones de Ica, Arequipa, Moquegua y Tacna (Vásquez 2009).

La pesquería de recursos bentónicos se caracteriza por ser una actividad extractiva, y de recolección en el caso de la varazón de algas pardas, con régimen de libre acceso. Esta actividad artesanal ha sufrido variantes en la modalidad de pesca, y las características de la flota, motivados principalmente por un fuerte incremento de la demanda de la industria exportadora por presiones internacionales de materia prima (Vásquez 2009).

Las principales especies de macroalgas que se extraen comercialmente en San Juan de Marcona son $M$. integrifolia (Sargazo) y $L$. trabeculata (Aracanto), las que se distribuyen en zonas inter y submareales someras de la zona marino costera. Si bien las principales zonas de distribución de estas macroalgas se encuentran al sur de Pta. San Juan, se han registrado importantes praderas en Pta. San Nicolás; así como, en zonas más distantes como San Fernando y Carro Caído al norte de San Juan de Marcona, como se aprecia en la Figura 12 (Vásquez 2009).

Durante los últimos años, respondiendo a las presiones crecientes de los mercados internacionales por materia prima para la extracción de ácido algínico, se ha incrementado significativamente la cosecha y colección de Lessonia spp. en el sur del Perú. La sobre explotación del recurso pone en riesgo dos aspectos muy importantes, el primero es que se está permitiendo que no haya una renovación apropiada de las praderas naturales y en segundo lugar se estaría afectando a las comunidades del macrobentos biológicamente delimitadas que se encuentran en los discos de fijación de estas algas. Es por este motivo que se planteó como objetivo general para este estudio determinar el efecto de la extracción del alga L. trabeculata sobre la riqueza y abundancia del macrobentos asociado al alga en la zona costera de San Juan de Marcona.

\section{Materiales y métodos.}

El estudio estuvo dirigido exclusivamente a la zona submareal del área comprendida entre Punta San

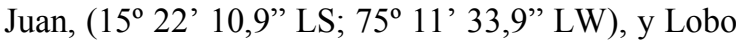
Fino (15 $24^{\circ} 44,7^{\prime \prime}$ LS; $75^{\circ} 08^{\prime} 18,7^{\prime \prime}$ LW), como se aprecia en la Figura 1. La colecta se realizó los días 26 y 28 de marzo del año 2012.

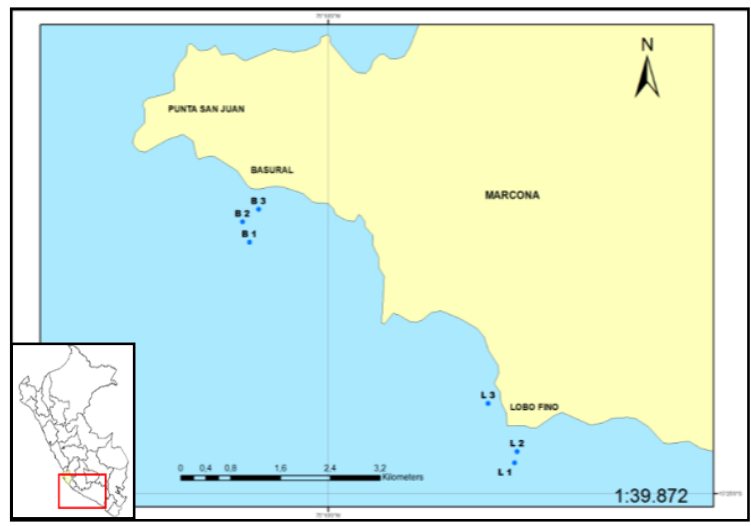

Figura 1. Ubicación del área de estudio. Fuente: IMARPE, 2012

Se realizó un muestreo estratificado sistemático, comprendiendo 02 sectores (Cuadro 1), en cada uno de los cuales se ubicaron 03 transectos con 03 estaciones (réplicas), distribuidos en intervalos de profundidad de: $5-10 \mathrm{~m}, 10-15 \mathrm{~m}$ y $15-20 \mathrm{~m}$. (Fig. 2).

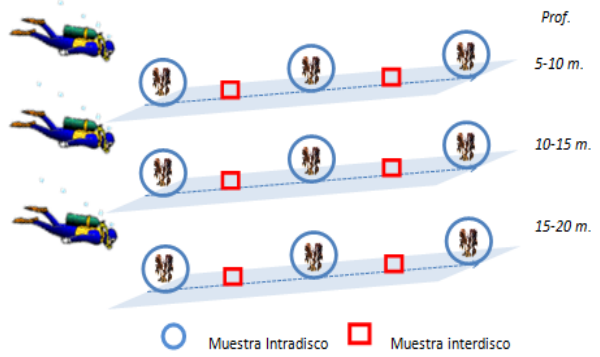

Figura 2. Diseño de Investigación.

2.1 Identificación y Caracterización de la diversidad y abundancia de macroinvertebrados asociados intra e interdisco.

La caracterización de la comunidad de macroinvertebrados asociados a los discos de adhesión (intra-disco) se realizó colectando plantas de L. trabeculata en las localidades seleccionadas aplicando algunas modificaciones a la metodología establecida por Vásquez et al. 2008.

Los discos de adhesión se colectaron mediante buceo semiautónomo desde los ambientes rocosos submareales con intervalos de profundidad de 5-10 $\mathrm{m}, 10-15 \mathrm{~m}$ y de 15 a $20 \mathrm{~m}$. En cada sitio de muestreo se extendió un transecto de 10 metros lineales paralelos a la línea de costa. En cada transecto se colocaron 3 circunferencias de $2 \mathrm{~m}^{2}$ de área separadas uniformemente. De cada unidad 
muestral se extrajo una planta al azar con diámetro basal entre 15 y $40 \mathrm{~cm}$.

Cada uno de los discos de adhesión ( $\mathrm{n}=3$ por estación) se despegaron del sustrato con barretas de fierro, previo corte de estipes y frondas por sobre la estructura del disco de adhesión. Luego, cada disco fue recubierto con una malla $(5 \mathrm{~mm}$ de apertura) para evitar el escape de los invertebrados móviles. Los discos de adhesión y la fauna asociada se depositaron en bolsas plásticas rotuladas y preservadas en formalina diluida al $7 \%$ en agua de mar, con la previa medición y pesado de los discos (Figuras 3 y 4 ).

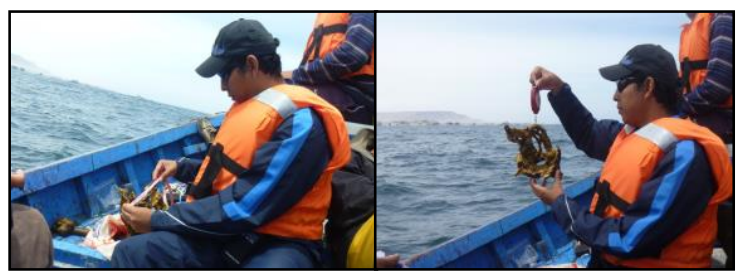

Figura 3. Medición y Pesado del disco de fijación luego de la extracción.

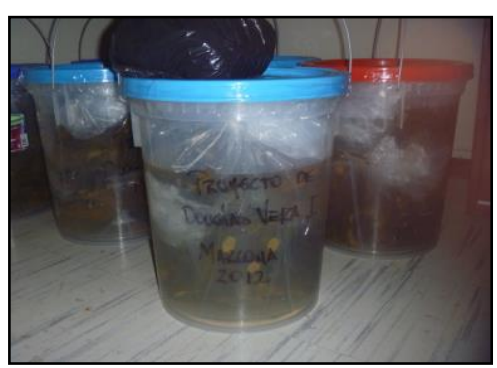

Figura 4. Muestras preservadas con formalina.

En el laboratorio, los organismos presentes en las cavidades interiores de los discos de adhesión de algas pardas fueron removidos por disección (Figura 5), de acuerdo a la metodología utilizada por Vásquez y Santelices (1984). Los individuos recolectados (> $5 \mathrm{~mm}$ ) fueron identificados hasta el nivel taxonómico más bajo posible (Vásquez et al. 2008).

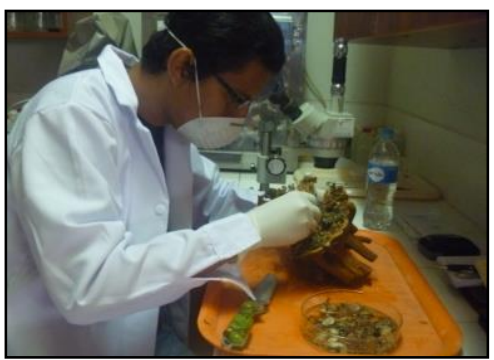

Figura 5. Proceso de disección y separación de organismos en el laboratorio.
La caracterización inter-disco se realizó sobre los mismos transectos utilizados anteriormente. Como unidades de muestreo se utilizaron cuadrantes de 50 $\mathrm{cm}$ por $50 \mathrm{~cm}$, abarcando un área de $0,25 \mathrm{~m}$. Se colectó la totalidad de organismos, dentro del cuadrante, los mismos que fueron preservados en formalina diluida al $7 \%$ en agua de mar e identificados en el laboratorio.

Se empleó bibliografía especializada de Álamo y Valdivieso (1987), Barnard (1954 y 1960), Chirichigno (1970), Fauchald (1977), Hobson y Banse (1981), Marincovich (1973), Paredes y Cardoso (2007), Lancelloti y Vásquez (2000), y Vásquez et al. (2001) para el análisis taxonómico.

\subsection{Análisis de Datos.}

Para monitorear el efecto de los cambios en el ambiente es necesario contar con información de la diversidad biológica. En tal sentido se utilizó la abundancia de las especies por estación de muestreo para calcular el índice de diversidad de Shannon y Wiener (H') y el índice de equidad o uniformidad (J') de Pielou e índice de dominancia de Simpson (IMARPE 2010).

Para determinar el grado de similitud entre estaciones y especies de se realizó un análisis multivariado de clasificación numérica, usando el método de clasificación jerárquica aglomerativa con media ponderada (grupo par no ponderado con promedio aritmético UPGMA) (Sneath y Sokal 1973) con el índice de Bray-Curtis.

Los datos obtenidos fueron tabulados y procesados en hojas de cálculo Excel V.2010 (Microsoft, Inc.) y PRIMER V.6,0 (Primer-E, Inc.).

\subsection{Evaluación de la Extracción de Lessonia trabeculata.}

Para realizar la evaluación de la extracción de éste recurso se tomaron en cuenta todas las Resoluciones Ministeriales emitidas por el Ministerio de la Producción vinculadas a las autorizaciones y prohibiciones emitidas por la entidad mencionada. Además se ha tomado como referencia el Anuario Estadístico de la Producción 2010 de PRODUCE.

\section{Resultados y Discusión.}

\subsection{Identificación de la diversidad del macrobentos.}

En el área muestreada se registraron un total de 5399 organismos agrupados en 71 especies distintas, 
tomando en cuenta las estaciones de Lobo Fino y Basural, de los cuales se lograron identificar 5369 organismos y 30 no identificados (Figura 6), correspondiendo 31 especies al Phylum Anélida, 17 a Mollusca, 15 a Crustácea, 4 a Equinodermata, 1 a Tunicata, 1 a Nemertea, 1 a Picnogónida y 1 a la Ictofauna del sector (Figura 7).

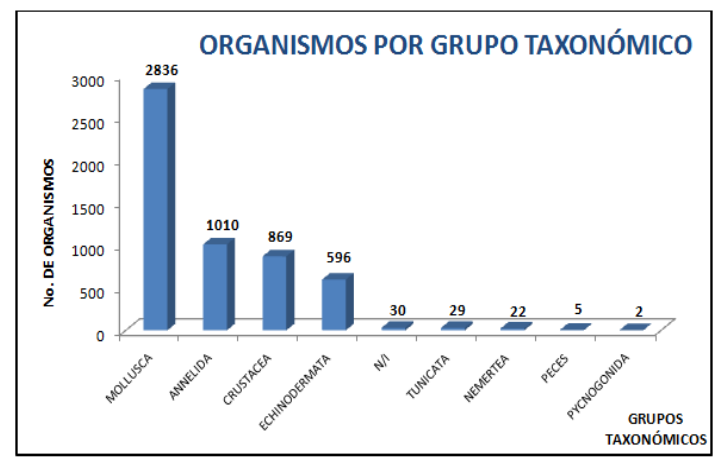

Figura 6. Número de organismos registrados por cada grupo taxonómico.

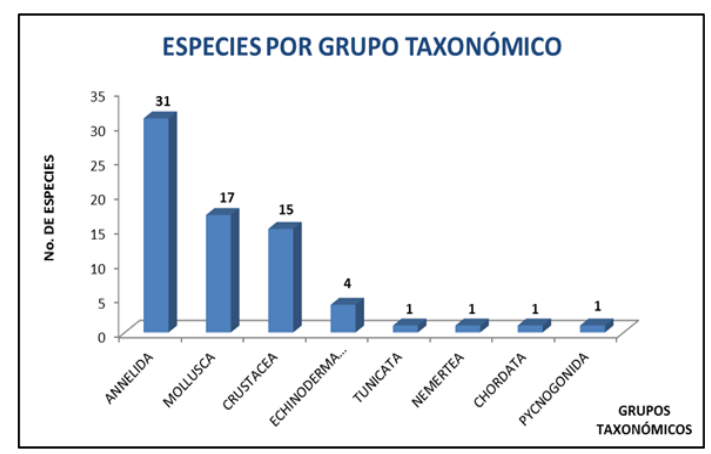

Figura 7. Número de especies registradas por cada grupo taxonómico.

Las especies más abundantes en ambas áreas investigadas fueron: Phragmatopoma moerchi, Syllis sp. y Scoletoma tetraura del Phylum Anélida, Brachidontes granulata, Crepipatella dilatata y Calyptraea trochiformis del Phylum Mollusca; Balanus laevis y Pachycheles crinimanus y del Phylum Crustácea y Ophiactis kroyeri y Tetrapigus niger del Phylum Echinodermata. Además, se observó escasa presencia de invertebrados de importancia comercial como Concholepas concholepas (loco) y Fissurella sp. (Lapa).

\subsection{Identificación de la diversidad del macrobentos.}

El Número de especies (S) varió entre 13 y 37 considerando las muestras intradisco, siendo las subestaciones P.3.2 y P.3.3 en Lobo Fino las de mayor número con $36 \quad$ y 37 especies respectivamente, mientras que para las muestras interdisco el Número de especies evidenciado vario de 8 a 24, registrando la mayor cantidad en la estación I.4 en la zona del Basural.

Respecto a la diversidad de especies aplicando el índice de Margalef (d) se apreció una variación entre 2,398 y 6,422, siendo la subestación P.1.1 la de menor diversidad y la P.3.3 la de mayor, con un promedio de 4,482. De las 18 subestaciones con muestras intradisco sólo 5 presentaron un índice igual o mayor a 5. En cuanto a las muestras interdisco, la estación I.4 presentó el mayor índice con 4,098 .

El índice de Shannon Wiener ( $\left.\mathrm{H}^{\prime}\right)$, que expresa la uniformidad de los valores de importancia a través de todas las especies de la muestra, reflejo como valor mínimo 1,528 correspondiente a la subestación P.1.1 y un mayor valor para la subestación P.3.3 con 2,69 .

En cuanto a la proporción de la diversidad observada con relación a la máxima diversidad esperada, que lo expresa el índice de Equidad de Pielou ( $\left.\mathrm{J}^{\prime}\right)$, se evidenció que la subestación P.1.1 tiene el valor más bajo con 0,5957 y P.6.2 el mayor valor con 0,7938 , valores para las muestras intradisco.

La dominancia de Simpson (D), que manifiesta la probabilidad de que dos individuos tomados al azar de una muestra sean de la misma especie, varió entre 0,6357 y 0,8958 con un promedio de 0,8310 para las muestras intradisco.

El análisis de clúster realizado con PRIMER 6.0 nos da como resultado que entre las subestaciones de El Basural existe una similaridad del $61 \%$ lográndose identificar tres grupos bien definidos (Figura 8), siendo la subestación B.4.3 la que se diferencia en un solo grupo por la mayor riqueza de especies de poliquetos.

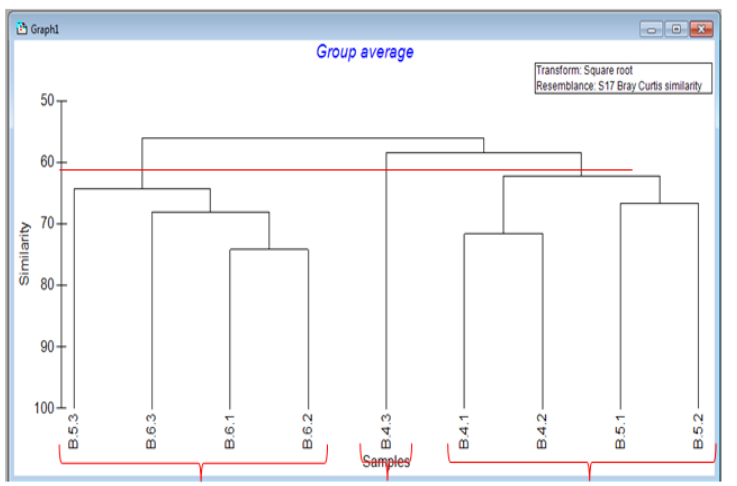

Figura 8. Análisis de Clúster realizado con las subestaciones frente a la Estación de El Basural. 
En la Figura 9 se puede observar que entre las subestaciones frente a la Estación de Lobo Fino a un $62 \%$ de similaridad se identifican cuatro grupos definidos. Las subestaciones L.1.1 y L.1.2 forman grupos separados por las diferencias existentes en la diversidad de moluscos y crustáceos, mientras que sobre los otros grupos que se forman la diferencia se da por la menor diversidad de especies de poliquetos.

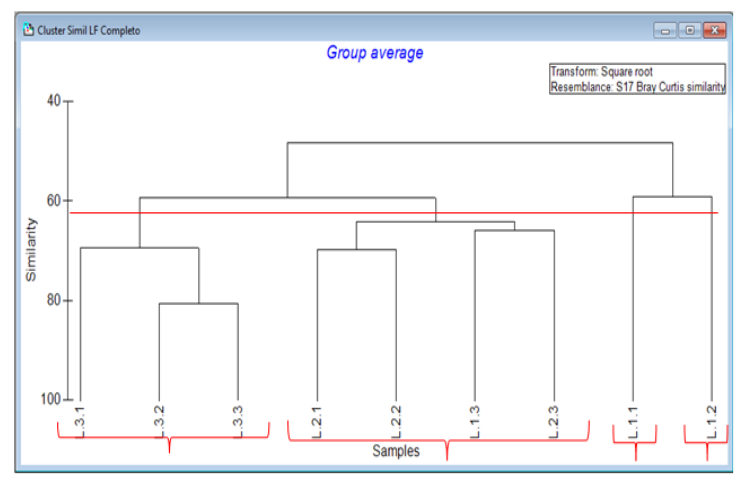

Figura 9. Análisis de Clúster realizado con las subestaciones frente a la Estación de Lobo Fino.

Al realizar el análisis de similaridad entre todas las subestaciones, tanto de las muestras intradisco e interdisco, de las Estaciones de El Basural y Lobo Fino se pueden observar tres grupos al $30 \%$ de similaridad (Figura 10). Revisando la base de datos se puede detectar que existe una menor riqueza de especies en las muestras interdisco.

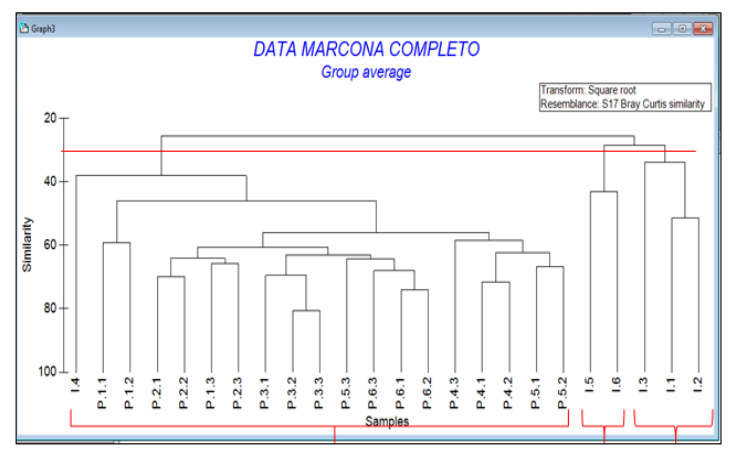

Figura 10. Análisis de Clúster realizado con todas las muestras de ambas estaciones estudiadas.

\subsection{Diámetro mayor del rizoide}

De las 09 muestras obtenidas en la zona de Lobo Fino se registraron 04 rizoides que tuvieron una medida por debajo de los $20 \mathrm{~cm}$. que establece la normativa para permitir su extracción correspondiendo al 44,4\%, mientras que en la zona del Basural, de las 09 muestras, sólo se registró un rizoide, el mismo que tuvo una medida de $15 \mathrm{~cm}$. y que corresponde al $11,1 \%$.

\section{Conclusiones.}

Se logró identificar el 99,4\% de la diversidad del macrobentos asociado a los discos de adhesión del alga $L$. trabeculata, registrándose en total 71 especies, lo que permite apoyar los criterios vertidos por otros investigadores acerca de la importancia ecológica fundamental que cumplen estos ecosistemas para las comunidades con las que se relacionan fuertemente.

La aplicación de los índices de abundancia, diversidad y uniformidad nos permitieron caracterizar las comunidades de macrobentos intradisco e interdisco. La diversidad intradisco determinada con el índice de Shannon no muestra diferencias significativas entre las subestaciones y se puede manifestar que existe una buena diversidad en las muestras analizadas debido a que el $89 \%$ de éstas presentan valores superiores a los 2 bits/ind, mientras que interdisco la diversidad se establece entre 1,29 bits/ind a 1,87 bits/ind.

La respectiva evaluación de la extracción de L. trabeculata registrada en el área de estudio nos permite determinar que en los últimos años si se han venido respetando las cuotas de extracción e incluso gracias a la iniciativa de los propios colectores no se han extraído las macroalgas, desde el año 2010, en la zona de Lobo Fino hasta Yaryarina con la finalidad de que exista la repoblación natural del recurso.

Se puede determinar, en base al tamaño de los discos de adhesión de los rizoides, que si existe una relación directa entre la extracción y el recurso L. trabeculata debido a que en la zona de Lobo Fino se evidencio una población más joven y en recuperación mientras que en El Basural ya existe una población madura con el $88,9 \%$ de las muestras registrando diámetros mayores a los $20 \mathrm{~cm}$. Esto podría afectar a la diversidad intradisco, en la medida que no exista este medio de protección para las especies pero los resultados de este estudio nos demuestran que en la zona que fue afectada presenta en la actualidad una menor abundancia de organismos aunque la riqueza de especies no varía significativamente.

\section{Referencias}

[1] Álamo, V. V.; Valdivieso, V. 1987. Lista sistemática de moluscos marinos del Perú. Boletín extraordinario Instituto del Mar del Perú. 205 p. 
[2] Barnard, J.L. 1954. Amphipoda of the family Ampeliscidae collected in the Eastern Pacific Ocean by the Velero III and Velero IV. Allan Hancock Pacific Expeditions 18 (1): 1-137.

[3] Barnard, J.L. 1960. The amphipod family Phoxocephalidae in the Eastern Pacific Ocean with analyses of other species and notes for revision of the family. Allan Hancock Pacific Expeditions 18 (3): 1-105.

[4] Cancino, J. M. \& Santelices, B. 1984. Importancia ecológica de los discos adhesivos de Lessonia nigrescens Bory (Phaeophyta) en Chile central. Revista Chilena de Historia Natural. 56: 2333.

[5] Chirichigno, N. 1970. Lista de Crustáceos del Perú (Decápoda y Stomatopoda). Instituto del Mar del Perú. Informe No. 35.

[6] Fauchald, K. 1977 The polychaete worms. Definitions and keys to the orders, families and genera. Natural History Museum of Los Angeles Country, Science Series 28: 1-188.

[7] Hobson, K.D.; Banse, K. 1981. Sedentariate and archiannelid polychaetes of British Columbia and Washington. Canadian Bulletin of Fisheries and Aquatic Sciences 209.

[8] IMARPE (Instituto del Mar del Perú). 2010. Informe: Evaluación poblacional de Lessonia trabeculata Villouta \& Santelices, 1986, entre El basural y Yaryarina, San Juan de Marcona. Unidad de Investigaciones en Biodiversidad. Laboratorio Costero de Pisco. 11 p.

[9] IMARPE (Instituto del Mar del Perú). 2012. Informe: Estudios sobre Macroalgas Pardas en el Sur del Perú 2011-2015. Volumen extraordinario. $200 \mathrm{p}$.

[10] Lancelloti, D. A. \& Vásquez, J. A. 2000. Zoogeografía de macroinvertebrados bentónicos de la costa de Chile: contribución para la conservación marina. Revista Chilena de Historia Natural. 73 (1):99-129.

[11] Marincovich L. 1973. Intertidal mollusk of Iquique, Chile. Natural History Museum Los Angeles County Science Bulletin 16: 1-49.

[12] Moore, PJ. 1971. The nematode fauna associated with holdfast of kelp (Laminaria hyperborea) in North East Britain. Journal of Marine Biology Association U.K. 51: 589-604

[13] Paredes C. \& Cardozo F. 2007. La Familia Calyptraeidae en el Perú (Gastropoda:
Caenogastropoda). Rev. Perú. biol. 13(3): 177 184. ISSN 1727-9933.

[14] Vásquez, J. A \& Santelices, B. 1984. Comunidades de macroinvertebrados en discos adhesivos de Lessonia nigrescens Bory (Phaeophyta) en Chile central. Revista Chilena de Historia Natural 57:131-154

[15] Vásquez, J. A. 2009. Informe Final del Estudio de Investigación de Poblaciones y de las condiciones de viabilidad ecológica de las actividades extractivas de algas pardas e invertebrados en la zona costera sur, en apoyo a la investigación y desarrollo del IMARPE. Proyecto UE-Perú/PENX. Lima, Perú. 90 p..

[16] Villouta, E. \& Santelices, B. 1986. Lessonia trabeculata sp. nov. (Laminariales, Phaeophyta), a new kelp from Chile. Phycologia 25:81-86. 
